\title{
Combined Method of Two Stage LPG-PCA Denoising with Impact on Preprocessing Step for Noisy Images
}

\author{
Jansirani S \\ Department of Computer \\ Science and Engineering \\ Jansons Institute of Technology \\ Coimbatore-641659
}

\author{
Karthikeyan S \\ II Year, Department of \\ Electronics and Communication \\ Engineering \\ Jansons Institute of Technology \\ Coimbatore-641659
}

\author{
Kiruba Priyadharsihni V \\ Department of Computer \\ Science and Engineering \\ Jansons Institute of Technology \\ Coimbatore-641659
}

\begin{abstract}
Image denoising plays a vital role in many image processing application to reduce the noise level without affecting the original image features. In this paper a powerful image denoising algorithm LPG-PGA with preprocessing step like Diffusion is introduced to improve the quality of the image. Per-processing step like diffusion is a necessary one in-case of noise is an important consideration, where the diffusion filter has strong smoothing characteristics [4-5]. PCA is statistical techniques which can be used to reduce the dataset from higher dimension to lower dimension without huge loss of image features [1]. The proposed system performance is evaluated by using various types of objective metrics like PSNR, SSIM, MSE, LMSE and NAE. The result shows that the proposed method has good promising performance compare to existing method.
\end{abstract}

\section{Keywords}

LPG-PCA denoising, Edge enhancing diffusion.

\section{INTRODUCTION}

Image processing is an active research area in many applications like Remote Sensing, Medical Imaging, Nondestructive Evaluation, Forensic Studies, Textiles, Material Science, Military, Film industry, Document processing, Graphic arts, Printing Industry, etc. Several image processing techniques are introduced to enhance the image quality by suppress the noise level [1-4]. Each method has its own merits and demerits. In this paper we combine two stage LPG-PCA method with preprocessing step (Diffusion) which is shown in Fig 1.

Image smoothing is a technique of improving the quality of an image by removing the noise in that image [7]. Among the several image processing technique we introduce LPG-PCA De-noising with preprocessing step to improve the quality of an image compare to existing methods. PCA is a decorrelation technique in statistical signal processing which can be used in several applications like pattern recognition, Dimensionality reduction, etc. In PCA the local statistics are calculated from the local PCA transformation matrix by using the moving window. In LPG-PCA the local statistics are computed in such a way that it preserves the edge structures of the image after the shrinkage in the PCA domain [1]. The diffusion process can be seen as an evolution process with an artificial time variable $t$ denoting the diffusion time where the input image is smoothed at a constant rate in all directions [6]. Linear Diffusion is a conventional way to smooth an image in a controlled way to convolve with a Gaussian kernel. The main drawback of linear diffusion method is, the smoothing process does not consider the information about important image features such as edges. It follows that same amount of smoothing to be applied at every image location. As a result, the diffusion process does smooth not only noise, but also image edges [6]. Hence we apply one of the Nonlinear diffusion method called edge enhancing diffusion which is discussed in section 3 .

\section{LPG-PGA BASED DE-NOISING}

Principle Component Analysis (PCA) is a method of identifying patterns in the data, and expressing the data in a way of highlight their similarities and differences. Since patterns in the data can be hard to find in the data of high dimension, where the luxury of graphical representation is not available, PCA is a powerful tool for analyzing data. The other main benefit of PCA is that once the patterns can be found it is easy to compress the data (i.e. by reducing the number of dimensions), without much loss of information [13].

Statistically, PCA is a de-correlation technique which is mainly used in pattern recognition, dimensionality reduction, etc. By transforming the original dataset into PCA domain and preserving only the several most significant principal components, the noise and trivial information can be removed. A PCA-based scheme was proposed for image de-noising by using a moving window to calculate the local statistics, from which the local PCA transformation matrix was estimated. PCA can be used to remove noise from images. PCA is a powerful statistical technique that is designed for determining lower dimensional representations of sampled data, which at first glance in its original representation may seem unstructured and random.

\section{DIFFUSION}

Recently several techniques are introduced to enhance the quality of an image. Among all these methods diffusion is one of the method used to enhance the quality of an image. In this method smoothing can be done by preserving the features of the original image. Among linear and non-linear diffusion here we propose a non-linear diffusion called edge enhancing diffusion which involves, constructing the diffusion tensor so that it mirrors the edge structure, thus preserving the edges by smoothing parallel to the edges. This allows smoothing near the edge, while preserving the edges themselves [4].

Edge enhancing diffusion constructs the diffusion tensor D as,

$$
D=R^{T}\left(\begin{array}{ll}
C_{1} & 0 \\
0 & C_{2}
\end{array}\right) R
$$




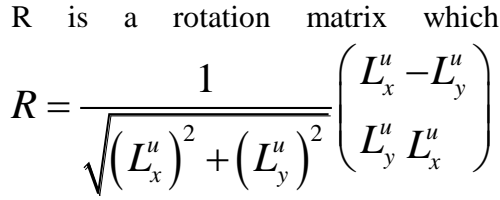

as,

Hence the D is calculated as,

$D=\frac{1}{\left(L_{x}^{u}\right)^{2}+\left(L_{y}^{u}\right)^{2}}\left(\begin{array}{ll}C_{1}\left(L_{x}^{u}\right)^{2}+C_{1}\left(L_{y}^{u}\right)^{2} & \left(c_{2}-c_{1}\right) L_{x}^{u} L_{y}^{u} \\ \left(c_{2}-c_{1}\right) L_{x}^{u} L_{y}^{u} & C_{1}\left(L_{y}^{u}\right)^{2}+C_{1}\left(L_{x}^{u}\right)^{2}\end{array}\right)$

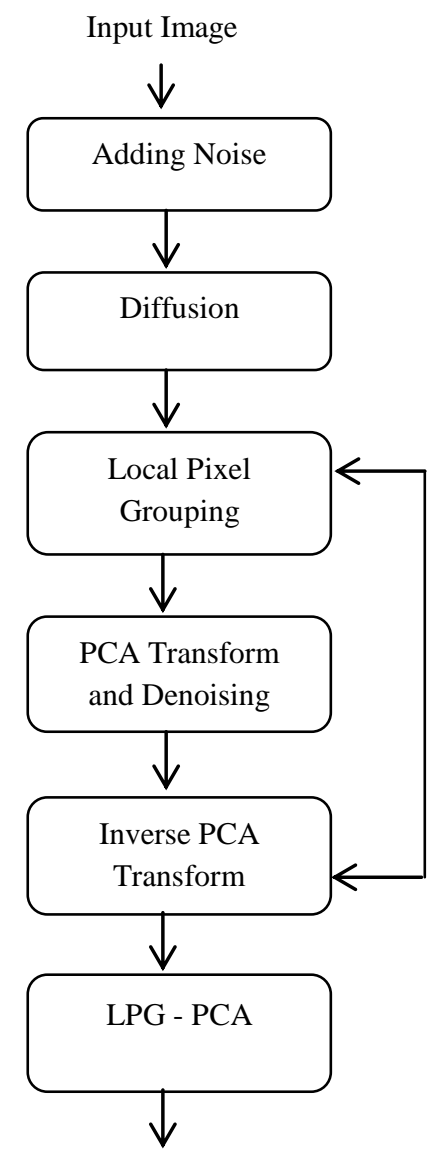

LPG - PGA

Output: Denoised Image

Fig 1: Two stage LPC-PGA Denoising with Diffusion

\section{IMAGE QUALITY MEASUREMENTS}

The various image quality metrics are used to discover the quality of the output image which are discussed below.

\subsection{Peak Signal to Noise Ratio (PSNR)}

$$
\begin{aligned}
& P S N R=10 \log _{10}\left(\frac{P e a k^{2}}{M S E}\right) \\
& P S N R=10 \log _{10}\left(\frac{255^{2}}{M S E}\right)
\end{aligned}
$$

PSNR is commonly expressed in terms of the logarithmic decibel scale. Higher PSNR value offers a good image quality.

\subsection{Structural Similarity Index Metrics (SSIM)}

$$
\operatorname{SSIM}=\frac{\left(2 \sigma_{x y}+C_{2}\right)\left(2 \times \bar{x} \times \bar{y}+C_{1}\right)}{\left(\sigma_{x}^{2}+\sigma_{y}^{2}+C_{2}\right)\left((\bar{x})^{2}+(\bar{y})^{2}+C_{1}\right)}
$$

Where $C_{1}$ and $C_{2}$ are constants.

$$
\bar{x}=\frac{1}{N} \sum_{i=1}^{N} x_{i} \quad \bar{y}=\frac{1}{N} \sum_{i=1}^{N} y_{i}
$$

The SSIM index is in the range between $[0,1]$. A value 0 indicates no correlation between the images. A value is close to one indicates that the denoised image is as close as to original image.

\subsection{Mean Squared Error}

$$
M S E=\frac{1}{M N} \sum_{i j}\left(A_{i j}-B_{i j}\right)^{2} \text {. }
$$

Where $A_{i j}$ is the original image, $B_{i j}$ is the denoised image. Larger the MSE value, image quality is poor.

\subsection{Laplacian Mean Squared Error (LMSE)}

$$
L M S E=\frac{\sum_{i=1}^{m} \sum_{j=1}^{n}\left(\nabla^{2} A-\nabla^{2} B\right)^{2}}{\sum_{i=1}^{m} \sum_{j=1}^{n}\left(\nabla^{2} A\right)^{2}}
$$

Smaller value of laplacian mean squared error provides good denoised image

\subsection{Normalized Absolute Error (NAE)}

$$
N A E=\frac{\sum_{i=1}^{m} \sum_{j=1}^{n}\left|\nabla^{2} A-\nabla^{2} B\right|}{\sum_{i=1}^{m} \sum_{j=1}^{n}\left|\nabla^{2} A\right|}
$$

Smaller value of Normalized Absolute Error (NAE) provides good denoised image.

\section{EXPERIMENTS AND DISCUSSION OF RESULTS}

The diffusion method discussed in section 3 were used to experimenting with a data base consists of various images. LPG-PCA and LPG-PCA along with diffusion method were applied to all images and their performance is compared. 


\subsection{Experiment with preprocessing step}

Preprocessing step so called diffusion is a method used for smoothing the image. The two anisotropic diffusion edge enhancing diffusion technique is proposed and the result is shown in Fig 2. Most of the PDE based methods are very crucial for Denoising and edge protective applications in images. While Perona and Malik types of nonlinear diffusion are isotropic, it preserves image features in the diffusion process by reducing dissipation in regions along image edges [11].

An advanced diffusion scheme was introduced by Weickert, called anisotropic diffusion. Among the two anisotropic diffusion edge enhancing diffusion is a Direction dependent diffusion method were strong diffusion is occurred along image edges, and weak diffusion is occurred across image edges. Edge Enhancing Diffusion Technique smooth's the original image.

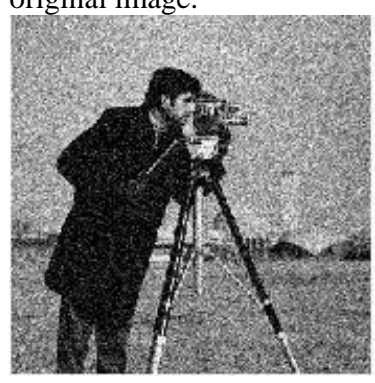

(a)

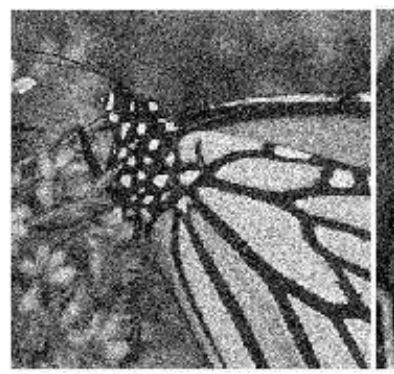

(c)

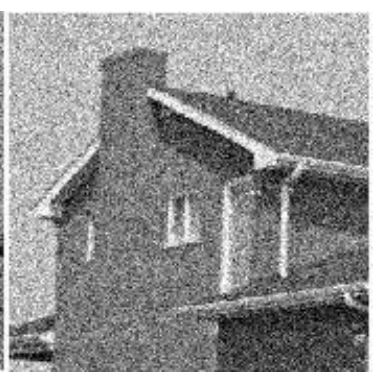

(b)

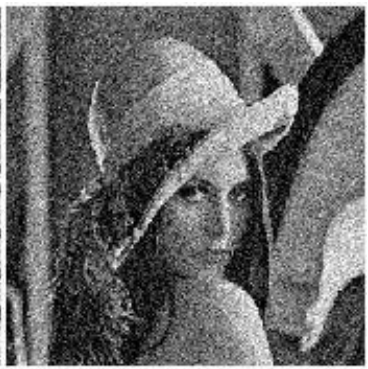

(d)
Fig 2: Illustrate the impact after pre-processing

\subsection{LPG-PCA with Diffusion Method}

The experiment is done by adding noise to the original image with various sigma values like 20,30 , and 40 . After that Preprocessing step like edge enhancing diffusion is applied to that noisy image and the result is shown in Fig 2. Then the two stage LPG-PCA denoising method is applied to that diffused image and the result is shown below. In Figure 3(d), 4(d), 5(d), 6(d), displays LPG-PCA denoising first stage result and Figure 3(e), 4(e), 5(e), 6(e), displays LPG-PCA denoising second stage result. In LPG-PCA De-noising, first stage removes most of the noise in the diffused image and second stage further improves the result of the first stage.

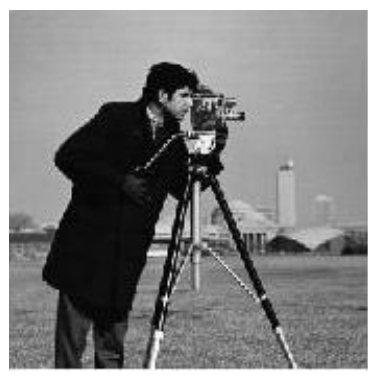

(a)

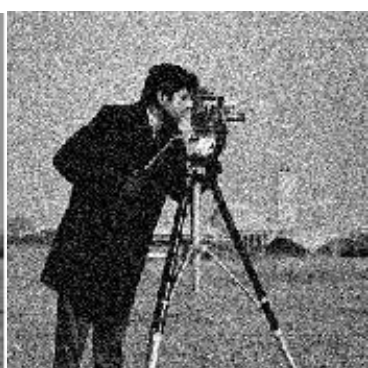

(b)

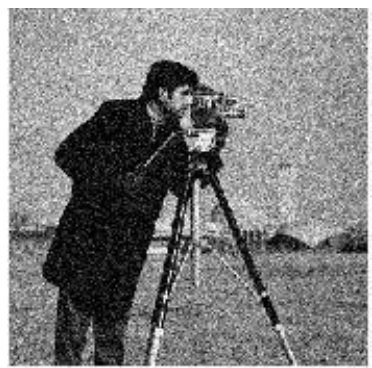

(c)

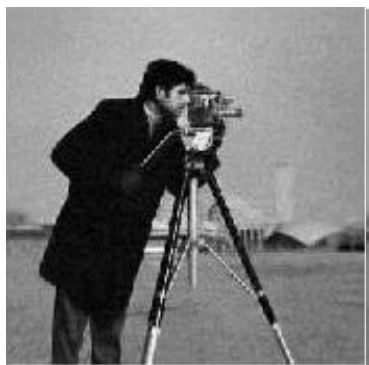

(d)

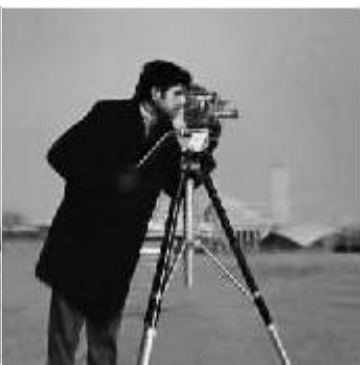

(e)
Fig 3: Illustrate the performance of proposed method with sigma $=30$. (a) Original image (b) Noisy image with sigma $=30$. (c) Diffused image (d) Denoised image after the first stage. (e) Denoised image after the second stage.

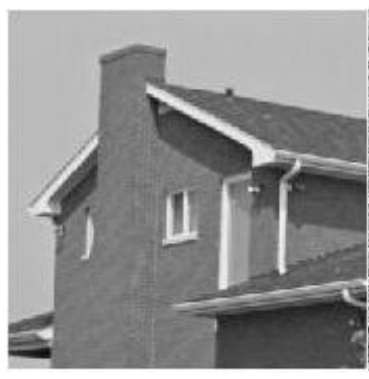

(a)

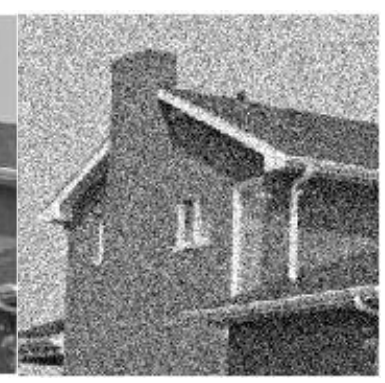

(b) 


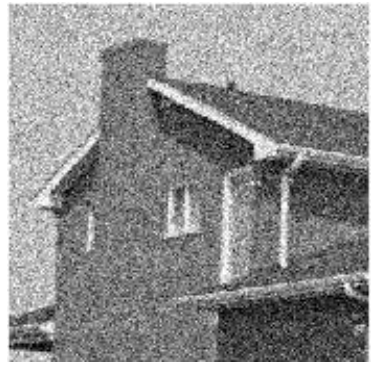

(c)

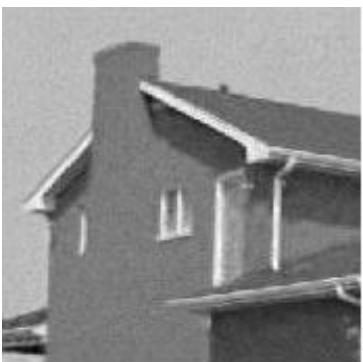

(d)

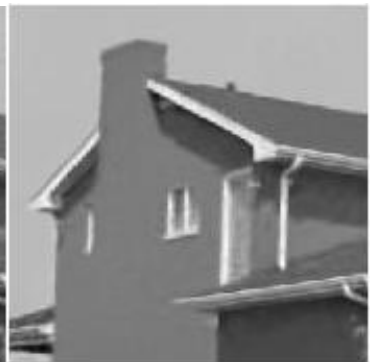

(e)
Fig 4: Illustrate the performance of proposed method with sigma $=30$. (a) Original image (b) Noisy image with sigma=30. (c) Diffused image (d) Denoised image after the first stage. (e) Denoised image after the second stage.

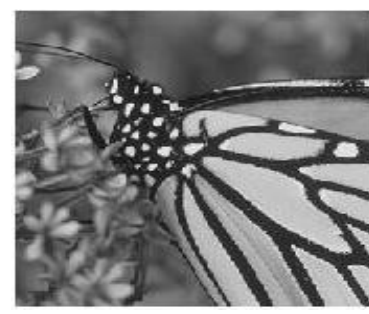

(a)

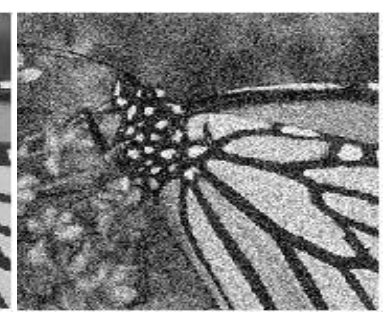

(b)

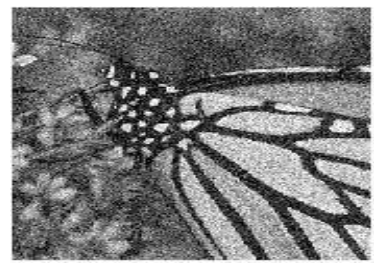

(c)

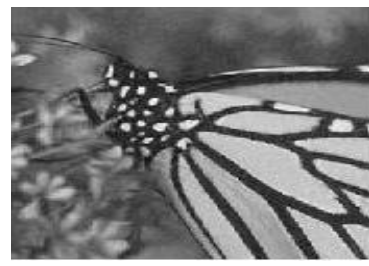

(d)

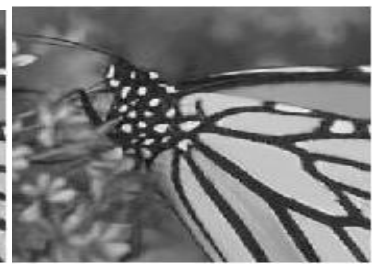

(e)
Fig 5: Illustrate the performance of proposed method with sigma $=30$. (a) Original image (b) Noisy image with sigma=30. (c) Diffused image (d) Denoised image after the first stage. (e) Denoised image after the second stage.

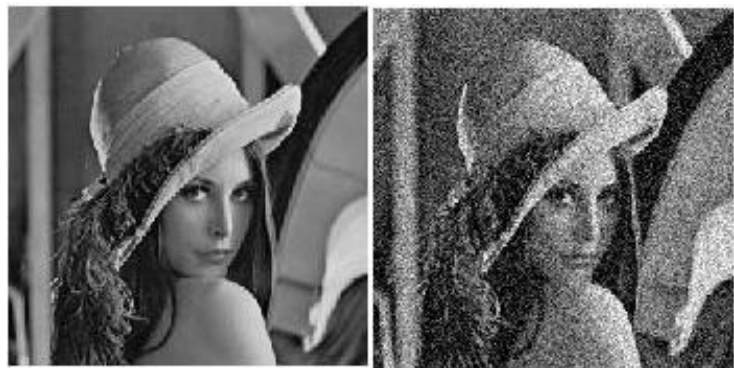

(a)

(b)

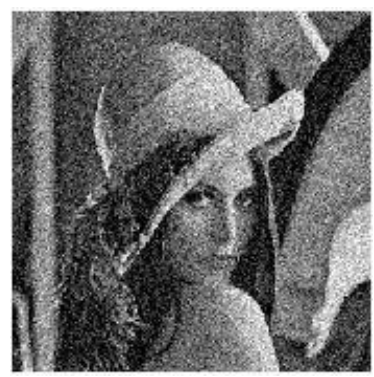

(c)

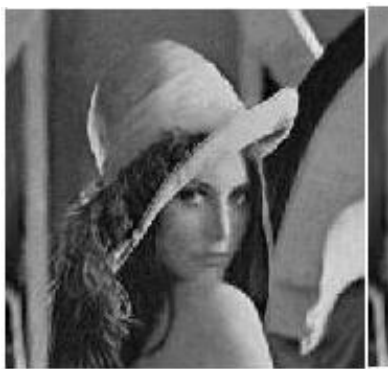

(d)

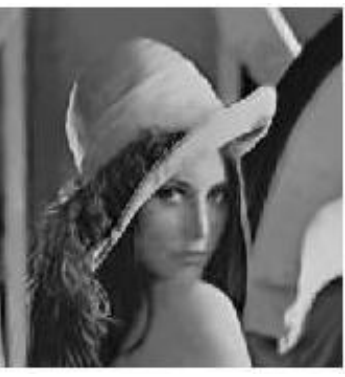

(e)
Fig 6: Illustrate the performance of proposed method with sigma $=30$. (a) Original image (b) Noisy image with sigma=30. (c) Diffused image (d) Denoised image after the first stage. (e) Denoised image after the second stage.

A total of two tables are illustrated to specify the performance of LPG-PCA and LPG-PCA with Diffusion method. Table 1 shows the precision obtained by the two stage LPG-PCA denoising method. Various image quality metrics like PSNR, SSIM, MSE, LMSE, NAE, are used for finding the quality of the image. The experiment is done with different sigma values. For each sigma value the image quality metrics are calculated for both stages. The result shows that PSNR and SSIM values are increased in stage 2 compare to stage 1 and MSE, LMSE, NAE values are decreased in stage 2 compare to stage 1 .Most of the noises are removed in stage 1 and stage 1 result is further improved in stage 2 . 
Table 1: Two stage LPG-PCA denoising method

\begin{tabular}{|c|c|c|c|c|c|c|c|c|c|c|c|}
\hline IMAGES & $\sigma$ & PSNR1 & PSNR2 & SSIM1 & SSIM2 & MSE1 & MSE2 & LMSE1 & LMSE2 & NAE1 & NAE2 \\
\hline \multirow[t]{3}{*}{1} & 20 & 32.2241 & 33.0828 & 0.8098 & 0.8677 & 38.9647 & 31.9743 & 0.9311 & 0.5092 & 0.0339 & 0.0281 \\
\hline & 30 & 29.7824 & 31.2095 & 0.7082 & 0.8393 & 68.3666 & 49.2187 & 1.6650 & 0.6364 & 0.0453 & 0.0341 \\
\hline & 40 & 27.8644 & 29.7322 & 0.6100 & 0.8124 & 106.3271 & 69.1613 & 2.6603 & 0.7464 & 0.0571 & 0.0400 \\
\hline \multirow{3}{*}{$\begin{array}{c}\text { 2) } \\
215\end{array}$} & 20 & 29.6746 & 30.0384 & 0.8779 & 0.9145 & 70. & 25 & 0.4963 & 0.3882 & 0.0539 & 0.0493 \\
\hline & 30 & 27.2477 & 27.7209 & 0.7989 & 0.8735 & 122.5483 & 109.8977 & 0.7636 & 0.5218 & 0.0720 & 0.0643 \\
\hline & 40 & 25.4704 & 26.0610 & 0.7185 & 0.8345 & 184.5184 & 161.0562 & 6694 & 0.6277 & 0.0894 & 0.0783 \\
\hline \multirow[t]{3}{*}{ 3) } & 20 & 30.2040 & 30.5415 & 0.8448 & 0.8765 & 62.0411 & 57.4018 & 0.5795 & 0.4770 & 0.0585 & 0.0540 \\
\hline & 30 & 27.8594 & 28.3595 & 0.7574 & 0.8292 & 106.4477 & 94.8703 & .8778 & 0.6250 & 0.0773 & 0.0691 \\
\hline & 40 & 26.1654 & 26.8566 & 0.6714 & 0.7882 & 157.2327 & 134.0965 & 1.2286 & 0.7289 & 0.0952 & 0.0825 \\
\hline \multirow[t]{3}{*}{ 4) } & 20 & 29.5114 & 29.7184 & 0.7980 & 0.8575 & 72.7683 & 69.3805 & 0.4088 & 0.3605 & 0.0502 & 0.0444 \\
\hline & 30 & 27.3693 & 27.8174 & 0.6927 & 0.8151 & 119.1662 & 107.4829 & 0.6189 & 0.4742 & 0.0653 & 0.0543 \\
\hline & 40 & 25.7708 & 26.4954 & 0.5978 & 0.7826 & 172.1858 & 145.7285 & 0.8609 & 0.5648 & 0.0795 & 0.0626 \\
\hline
\end{tabular}

Table 2: Two stage LPG-PCA denoising with Diffusion

\begin{tabular}{|c|c|c|c|c|c|c|c|c|c|c|c|}
\hline IMAGES & $\sigma$ & PSNR1 & PSNR2 & SSIM1 & SSIM2 & MSE1 & MSE2 & LMSE1 & LMSE2 & NAE1 & NAE2 \\
\hline 1) & 20 & 32.4854 & 33.0838 & 0.8258 & 0.8669 & 36.6890 & 31.9667 & 0.7701 & 0.5025 & 0.0325 & 0.0280 \\
\hline \multirow{2}{*}{$=\quad$} & 30 & 29.9675 & 31.2390 & 0.7223 & 0.8407 & 65.5137 & 48.8855 & 1.4711 & 0.6200 & 0.0441 & 0.0339 \\
\hline & 40 & 27.9917 & 29.7592 & 0.6202 & 0.8144 & 103.2539 & 68.7323 & 2.4523 & 0.7274 & 0.0561 & 0.0397 \\
\hline 2) & 20 & 29.8240 & 30.0707 & 0.8884 & 0.9164 & 67.7138 & 63.9750 & 0.4489 & 0.3796 & 0.0524 & 0.0488 \\
\hline \multirow{2}{*}{63} & 30 & 27.3481 & 27.7481 & 0.8085 & 0.8758 & 119.7484 & 109.2123 & 0.7102 & 0.5124 & 0.0707 & 0.0638 \\
\hline & 40 & 25.5436 & 26.0869 & 0.7260 & 0.8368 & 181.4341 & 160.1008 & 1.0158 & 0.6192 & 0.0883 & 0.0778 \\
\hline \multirow[t]{3}{*}{ 3) } & 20 & 30.3561 & 30.5660 & 0.8557 & 0.8778 & 59.9059 & 57.0794 & 0.5328 & 0.4751 & 0.0569 & 0.0535 \\
\hline & 30 & 27.9566 & 28.3772 & 0.7669 & 0.8312 & 104.0934 & 94.4852 & 0.8232 & 0.6201 & 0.0760 & 0.0686 \\
\hline & 40 & 26.2365 & 26.8750 & 0.6788 & 0.7903 & 154.6791 & 133.5290 & 1.1706 & 0.7227 & 0.0941 & 0.0821 \\
\hline \multirow[t]{3}{*}{ 4) } & 20 & 29.6252 & 29.7841 & 0.8146 & 0.8572 & 70.8863 & 69.9316 & 0.3843 & 0.3667 & 0.0486 & 0.0442 \\
\hline & 30 & 27.4570 & 27.8182 & 0.7063 & 0.8167 & 116.7832 & 107.6368 & 0.5873 & 0.4757 & 0.0639 & 0.0540 \\
\hline & 40 & 25.8403 & 26.5001 & 0.6071 & 0.7849 & 169.4535 & 145.5693 & 0.8258 & 0.5642 & 0.0784 & 0.0624 \\
\hline
\end{tabular}

Table 2 shows the precision obtained by the two stage LPGPCA Denoising method with preprocessing step. In table 2 PSNR1, PSNR2, SSIM1, SSIM2 values are increased and
MSE1, MSE2, LMSE1, LMSE2, NAE1, NAE2 values are decreased compare to table1. After preprocessing Two stage LPG-PCA denoising technique is applied and the result shows 
that the performance is improved compare to existing Two stage LPG-PCA denoising method.

\section{CONCLUSIONS}

LPG-PCA method along with Pre-processing step like Diffusion is used and the performance is validated through various performance metrics like PSNR, SSIM, MAE, LMSE, NAE and the result shows that our proposed method Twostage image denoising by principal component analysis, local pixel grouping with Diffusion method has higher accuracy compare to Existing method Two-stage image de-noising by principal component analysis with local pixel grouping.

\section{REFERENCES}

[1] Lei Zhang, Weisheng Dong, David Zhang, and Guangming Shi, "Two-stage image denoising by principal component analysis with local pixel grouping", Elsevier, Pattern Recognition 43 (2010), 1531-1549.

[2] K. John Peter, Dr K. Senthamarai Kannan, Dr S. Arumugan, and G.Nagarajan, "Two-stage image denoising by Principal Component Analysis with Self Similarity pixel Strategy", International Journal of Computer Science and Network Security, VOL.11 No.5, May 2011.

[3] Sabita Pal, Rina Mahakud, and Madhusmita Sahoo, "PCA based Image Denoising using LPG", IJCA Special Issue on "2nd National Conference- Computing, Communication and Sensor Network", 2011.

[4] Ahmed Badawi, J. Michael Johnson, and Mohamed Mahfouz Scatterer Density in Edge and Coherence Enhancing Nonlinear Anisotropic Diffusion for Medical Ultrasound Speckle Reduction, International Journal of Biological and Life Sciences, 2007.

[5] Joachim Weickert, Anisotropic Diffusion in Image Processing, Department of Computer Science University of Copenhagen Copenhagen, Denmark.

[6] Erkut Erdem, Linear Diffusion, Hacettepe University, February 24th, 2012
[7] Aditya Goyal, Akhilesh Bijalwan, Mr. Kuntal Chowdhury, A Comprehensive Review of Image Smoothing Techniques, International Journal of Advanced Research in Computer Engineering \& Technology, Volume 1, Issue 4, June 2012.

[8] Haixia Wang, Qian Kemao, Wenjing Gao, Feng Lin, Hock Soon Seah, " Partial Differential Equation Based Coherence Enhancing Denoising for Fringe Patterns", International Conference on Experimental Mechanics 2008, Vol. 7375, 2008.

[9] Weickert J., "Coherence-Enhancing Diffusion Filtering", International Journal of Computer Vision", vol. 31, issue 2-3, pp.111 - 127 (1999).

[10] Weickert J., V. Hlavac and R. Sara, Eds, "Multiscale Texture Enhancement", Computer analysis of images and patterns, Lecture Notes in Comp.Science, 970, Springer, Berlin, pp. 230-236(1995).

[11] Asha Ashok, Dhivya S, Jansirani S, K. P. Soman, Combined Method of Level set with impact on Pre-processing for binarization of document images in Tamil Script, IJCA Journal, Volume 48, 2012.

[12] L. Zhang, B. Paul, X. Wu, Hybrid inter- and intrawavelet scale image restoration, Pattern Recognition 36 (8) (2003) 1737-1746.

[13] D. Barash, A fundamental relationship between bilateral filtering, adaptive smoothing, and the nonlinear diffusion equation, IEEE Transaction on Pattern Analysis and Machine Intelligence, 24 (6) (2002) 844-847.

[14] D.D. Muresan, T.W. Parks, Adaptive principal components and image denoising, in: Proceedings of the 2003 International Conference on Image Processing, 1417 September, vol. 1, 2003, pp. I101-I104. [22]

[15] Z. Wang, A.C. Bovik, H.R. Sheikh, E.P. Simoncelli, Image quality assessment: from error visibility to structural similarity, IEEE Transaction on Image Processing 13 (4) (2004). 